Aus dem allgemeinen Krankenhaus Hamburg-Eppendorf, II. medizin. Abteilung (Oberarzt Dr. Nonne).

\title{
Weitere Beiträge zur Kenntnis des Kompressionssyndroms im Liquor cerebrospinalis.
}

Von

\author{
Dr. Wilhelm Raven, \\ Assistenzarzt.
}

Im vorigen Jahre habe ich in dieser Zeitschrift*) über die Bedeutung der Eiweissvermehrung und der häufig in Verbindung mit ihr auftretenden Xanthochromie der Lumbalflüssigkeit bei Rückenmarkskompression berichtet. Inzwischen sind von anderer Seite mehrere einschlägige Arbeiten erschienen und ich selbst hatte Gelegenheit, im Eppendorfer Krankenhaus auf der Abteilung meines Chefs, Herrn Oberarzt Dr. Nonne, 7 weitere Fälle zu beobacbten, die das Kompressionssyndrom mehr oder weniger ausgesprochen zeigten.

Über diese Fälle möchte ich kurz berichten, um mich dann der Frage zuzuwenden, wann isolierte Eiweissvermehrung, wann ausserdem Xanthochromie mit oder ohne Zellvermehrung entsteht. Wenn sich auch bisher auf Grund des Liquorbefundes allein keine Angaben über Art und Ausgangspunkt der Schädigung machen lassen, so ist doch zu hoffen, dass man allmählich für die verschiedenen Kompressionsursachen charakteristische Unterschiede in der Liquorbeschaffenheit wird feststellen können.

Ich lasse zunächst an die früher veröffentlichten 15 Fälle anschliessend die Krankengeschichten der neuen Fälle im Auszuge folgen.

Fal1 16. W. W. (Prot.-Nr. 13181/1912), 39jähriger Mann, bisher völlig gesund, erkrankte akut an kurzdauernden, periodisch auftretenden Parästhesien im linken Arm, die allmählich an Intensität zunahmen. Nach

*) 1912. Bd. 44, S. 380 . 
zwei Monaten traten dumpfe, "rheumatische" Schmerzen im Nacken auf, anschliessend Druckempfindlichkeit in Höhe des 5. Halswirbels und nach leichtem Kópftrauma geringe Schwäche und taubes Gefühl in den Fingern der linken Hand.

In einer auswärtigen Knlinik wurde auf Grund des Röntgenbildes ein destruierender Prozess des 6. Halswirbels angenommen.

Status: Grosser, kräftiger Mann. Innere Organe gesund.

Bei Kopfbeugung nach vorn und hinten Schmerzen, keine Krepitation. Am Hals Druckempfindlichkeit zu beiden Seiten des 5. Dornfortsatzes, besonders rechts.

Sehnenreflexe links lebhafter, als rechts.

Fuss- und Patellarklonus beiderseits.

Babinski links + .

Haut- und Schleimhautreflexe normal.

Leichte motorische Schwäche des linken Armes.

Hypästhesie für alle Qualitäten in demselben Arm, Astereognosie des Vorderarms, leichte Ataxie.

Elektrische Erregbarkeit durchweg normaj.

Die auswärts vorgenommene Lumbalpunktion hatte ergeben: Klarer Liquor, Druck $240 \mathrm{~mm}$.

Phase $\mathrm{I}++$.

Nissl $91 / 2$ Strich!

Wassermann im Blut $\emptyset$.

Die nochmalige Röntgenaufnahme liess ausser einer leichten Ostitis deformans der Halswirbelsäule kejne Veränderungen erkennen.

Es wurde eine Kompression des Rückenmarks, wahrscheinlich durch Tumor, angenommen und sogleich zur Laminektomie in Höhe des 5. und 6. Halswirbels geschritten. Dabei wurde auch an der gesuchten Stelle der Tumor gefunden, der links hinten und in der Mitte auf dem Rückenmark lag. Leider zeigte sich, dass er aus dem Innern des Rückenmarks herausgewachsen war, dieses diffus infiltrierend.

Exitus am folgenden Tage.

Die Sektion ergab, dass es sich um ein intramedulläres zellreiches Gliosarkom handelte, das sich durch das ganze Halsmark erstreckte und besonders die Hinterstränge und den linken Seitenstrang befallen hatte. An der Operationsstelle war es zapfenförmig nach hinten aus der Medulla herausgew achsen.

Fall 17*). A. S. (Prot.-Nr. 21731/1912), 38jährige, früher stets gesunde Frau, klagt seit 5 Wochen über "Ziehen" im ganzen Körper, Schmerzen beim Gehen im rechten Arm und Bein, in Schulter und Rücken.

Status: Mittelgrosse, kräftige Frau ohne krankhafte Veränderungen der inneren Organe, doch ist in der rechten Unterbauchgegend an der Darmbeinschaufel eine Geschwulst fühlbar.

Muskulatur des rechten Arms druckschmerzhaft, Motilität im Ellbogen und der Hand deutlich vermindert.

*) Die Erlaubnis zar Veröffentlichung dieses Falles verdanke ich Herrn Oberarzt Dr. Reiche, dem ich hierfür auch an dieser Stelle meinen aufrichtigen Dank aussprechen möchte. 
Schmerzen im rechten Hüftgelenk bei Bewegungen.

Reflexe sämtlich intakt.

Keine Sensibilitätsstörungen.

Nach 3 Wochen tritt plötzlich bei erhaltenen Reflexen Gefühllosigkeit der Beine auf. Am nächsten Tage besteht eine schlaffe Lăhmung beider Beine.

Patellar- und Achillesreflex beiderseits $\emptyset$.

Bauchdecken-, Sohlenreflexe ø.

Kein Babinski.

Stuhl- and Urinverhaltung.

Totale Anästhesie bis zur 3. Rippe hinauf.

Lumbalpunktion: Liquor klar, farblos. Drack $60 \mathrm{~mm}$.

Phase I t.

Lymphocytose $23 / 3$.

Wassermann $0,2-1,0$ ø.

Wassermann im Blut $\emptyset$.

Nach wenigen Tagen Exitus letalis.

Sektion: Apfelgrosses Sarkom des rechten Ovariums mit Metastasen in der Schilddrüse und der rechten Niere, sowie im 6. Halswirbel.

Auf der rechten Halsseite, im Bereich der Mm. longus colli et capitis, sowie des Scalenus posterior ist die Muskulatur von trübglänzenden grauweissen Geschwulstmassen durchsetzt. Die Wirbelsäule zeigt hier auf dem Durchschnitt am 6. Halswirbel eine gelbgraue, durch Tumormassen bedingte Farbe des ganzen Wirbelkörpers, dessen Konsistenz herabgesetzt ist. Auch der zugehörige Wirbelbogen zeigt die gelbgraue Färbnng. Ziemlich weiche, rosagraue Gewebsmassen sind von der oben beschriebenen Muskulatur weiter nach dem Wirbelkanal vorgedrungen und drücken in der Gegend des 6. Halswirbels auf das Rückenmark durch Abheben des Lig. longitudinale post. vom 6. Halswirbelkörper. Das Halsmark lässt auf der Schnittfläche sonst nur normale Zeichnung erkennen, ist in Höhe des 6. Halswirbels die Konsistenz herabgesetzt und auf dem Querschnitt die Zeichnung nicht mehr zu erkennen.

Fall 18. J. H. (Prot.-Nr. 21141/1912), 37jähriger, immer gesunder Mann. Seit 2 Monaten Schmerzen zwischen den Schulterblättern, ins rechte Bein ausstrahlend. Vor wenigen Tagen entstand über Nacht eine völlige Lähmung beider Beine mit Gefühllosigkeit. Am nächsten Tage Stuhl- und Urinverhaltung, Schwäche der Bauchmuskulatur und Abnahme des Gefühls am Leib.

Status: Kräftiger, normal gebauter Mann.

Bei Kopfbeugung Schmerzen im Rücken. Gegend des 2. bis 4. Brustwirbeldorns druck- und klopfempfindlich, hier auch leichter Gibbus.

Abdomen aufgebläht, Bauchdecken paretisch, Blase gespannt.

Totale schlaffe Lähmung beider Beine mit Areflexie.

Von der Mamillarlinie abwärts für alle Qualitäten hochgradige Hypästhesie, von der Mitte der Waden abwärts völlige Anästhesie.

Mastdarm- und Detrusorlähmung.

Nach wenigen Tagen tritt der Exitus ein.

I. Lumbalpunktion an gewöhnlicher Stelle, 1/2 Stunde post mortem: Geringer Druck, Liquor intensiv bernsteingelb, klar. 
Phase $\mathrm{I}+t+$

Lymphocytose: Im Präparat Nester von auffallend grossen, blassen, rundlichen Zellen mit blassem Kern.

Wassermann $0,2-1,0 \varnothing$.

Wassermann im Blut $\emptyset$.

II. Lumbalpunktion zwischen 7. Hals- und 1. Brustwirbeldorn: Liquor.

Unter sehr geringem Druck wenige Tropfen klarer, farbloser

Sektion: Bronchialcarcinomknoten im Oberlappen der rechten Lunge mit Metastasen in den Hilusdrüsen und der Leber, sowie im 4. Brustwirbelbogen. Der Bogen des 4. Brustwirbels ist auf der linken Seite ca. haselnussgross von einer weissen Tumormasse durchsetzt, die teilweise die benachbarten Muskeln derb infiltriert. Der Tumor erstreckt sich unter dem Lig. longitudinale posticum, dies stellenweise durchwachsend, bis zum 5. Brustwirbel und greift in dieser Ausdehnung auf die Aussenfläche der Dura über, die dadurch nach innen vorgewölbt wird.

Die mikroskopische Untersuchung des Rückenmarks ist noch nicht abgeschlossen.

Fall 19. F. G. (Prot.-Nr. 24520/1912), 53jähriger Maler. Eine Schwester ist jung an Phthise gestorben, sonst belanglose Anamnese. Vor $1 \frac{1}{2}$ Jahren vorubergehend "taubes" Gefühl im rechten Fuss, das vor einem Jahr wieder auftrat. Ausserdem kam jetzt eine gewisse Schwäche beider Beine hinzu, rechts $\mathrm{mehr}$ als links. In den letzten 2 Monaten haben die Erscheinungen zugenommen, Stuhl- und Urinentleerung sind erschwert.

Status: Untersetzter, muskulöser Mann.

Innere Organe, speziell die Lungen, gesund.

Wirbelsäule nirgends klopf- oder druckempfindlich.

Zwischen 4. und 7. Brustwirbel leichte Vorwölbung der Wirbelsäule nach hinten.

Bauchmuskulatur paretisch.

In beiden Beinen hochgradige Schwäche fast aller Bewegungen, rechts mehr als links.

Patellar- und Achillesreflex beiderseits lebhaft, aber kein Klonus.

Bauchdecken-, Kremaster-, Sohlenreflex beiderseits $\emptyset$.

Babinski beiderseits deutlich positiv.

Sensibilität: Abwärts von dem Proc. xiphoides für die einzelnen Qualitäten in verschiedener Stärke gestört, durchweg links hochgradiger, als rechts.

Lumbalpunktion: Normaler Druck.

Liquor klar, farblos.

Phase I ++ , sofort deutlich.

Lymphocy tose $\emptyset$.

Wassermann $0,2-1,00$.

Wassermann im Blut $\emptyset$.

Pirquet: Nach 18 Stunden schwach, nach 3 Tagen stark positiv.

Das Röntgenbild ergab eine Verschmälerung des 5. und 6. Brustwirbels und zu beiden Seiten einen gleichmässig scharf begrenzten, konvexen Schatten. 
Die Diagnose wurde auf tuberkulöse Spondylitis gestellt.

Unter konservativer Therapie (Kopfextension) gingen allmählich die Symptome zurück.

Fall 20. H. M. (Prot.-Nr. 22371/1912), 30jähriger Mann. Beide Eltern sind an Lungenleiden gestorben, jedoch soll es sich nicht um Tuberkulose gehandelt haben. Patient selbst war immer gesund, doch habe er seit 6 Jahren, ohne irgendwelche subjektiven Beschwerden, einen Buckel bekommen. Seit einigen Wochen bestehen Parästhesien in den Füssen, „als wenn sie eingeschlafen wären". Allmählich stieg dies Gefühl bis zur Leistenbeuge auf, auch konnte er im Liegen nicht mehr Wasser lassen.

Status: Gut genährter, kräftiger Mann. Innere Organe, auch die Lungen, gesund.

Vom 8. bis 11. Brustwirbel winkliger, nicht druckempfindlicher Gibbus.

Motilität überall intakt.

Bauchdeckenreflexe: Oberer and mittlerer lebhaft, unterer beiderseits schwach.

Sohlenreflex beiderseits $\emptyset$.

Patellar- und Achillesreflex rechts gesteigert.

Andeutung von Fussklonus rechts.

Babinski rechts positiv.

Leichte Hypalgesie am linken Bein und der linken Bauchseite bis zur Nabelhöhe.

Stereognosie, Lagegefühl erhalten.

Pirquet: + .

Röntgenaufnahme der Wirbelsäule: Flaschenförmiger Senkungsabszess, vom Gibbus ausgehend. Eine kariöse Einschmelzung der Wirbel ist nicht sicher nachweisbar.

Lumbalpunktion: Normaler Druck.

Liquor klar, farblos.

Phase $\mathrm{I}+\mathrm{t}$.

Lymphocytose $\emptyset$.

Wassermann $0,2-1,0 \quad 0$.

Wassermann im Blut $\emptyset$.

Unter Extensionsbehandlung subjektive und objektive Besserung.

Fall 21. S. M. (Prot.-Nr. 1725/1912), 41jährige Frau, belanglose Anamnese. Vor 5 Jahren Amputation der linken Mamma wegen Carcinom. Seit 2 Jahren unter Schmerzen in Beinen und Rücken allmähliche Kyphosenbildung, zunehmende Schwäche beider Beine bis zu völliger Lähmung.

Status: Sehr kachektische Frau mit gesunden inneren Organen. Schwere Morphinistin.

Hochgradige Kyphose der Wirbelsäule, am 6. Brustwirbel beginnend; am 11. bis 12. am stärksten ausgesprochen, dann schnell abfallend.

Urininkontinenz.

Bauchdeckenreflexe lebhaft.

Sohlenreflexe beiderseits $\emptyset$.

Patellarreflexe $\emptyset$.

Achillesreflexe rechts $\emptyset$, links + .

Kein Babinski.

Fast völlige Lähmung beider Beine, Reste von Motilität in den Zehengelenken. Muskulatur hochgradig atrophisch. 
Totale Anästhesie an beiden Unterschenkeln mit Ausnahme eines schmalen Streifens an der Aussenseite des linken Unterschenkels.

Leichte Hypästhesie für alle Qualitäten an der Hinterseite der Oberschenkel.

Lokalisation und Lagegefühl an den Füssen erloschen.

Lumbalpunktion: Druck $130 \mathrm{~mm}$.

Liquor leicht gelblich gefärbt.

Phase I +++ .

Lymphocytose $\emptyset$.

Bei längerem Stehen bildet sich ein weisses Netz im Liquor.

Nach monatelangem Verlauf, wobei ständig die heftigsten Schmerzen bestanden, trat schliesslich infolge totaler Blasen-Mastdarmlähmung Decubitus auf, der unter zunehmender Kachexie zum Exitus führte.

Sektion: Im Bereiche des 11. und 12. Brust- und des 1. Lendenwirbels ist die Wirbelsäule nach hinten konvex abgeknickt. Beim Einschneiden von hinten finden sich die Umgebung der genannten Wirbel und diese selbst durchsetzt von derben, gelblichgrauen Tumormassen, die bis auf die Dura reichen und zum Teil fest mit ihr zusammenhängen. Auf die Substanz des Rückenmarks dagegen haben sie nirgends übergegriffen. Die Medulla ist über der Knickungsstelle der Wirbelsäule stark gespannt und abgeplattet. Der 11. Brust- bis 1. Lendenwirbelkörper sind von Tumormassen so stark durchwuchert, dass nur noch geringe Knochenreste $\mathrm{zu}$ erkennen sind. Die histologische Untersuchung zeigte, dass es sich um Metastasen des vor 5 Jahren amputierten Mammacarcinoms handelte.

Fall 22. H. F. (Prot.-Nr. 18265/1912), 34jähriger, bisher stets gesunder Kutscher. Seit 6 Wochen erschwerte Urinentleerung, Schmerzen im Rücken und der Magengegend, seit 8 Tagen Lähmung der linken Gesichtsseite und beider Beine. Stuhlgang angehalten.

Status: Kräftiger Knochenbau, innere Organe intakt.

Facialisparese links.

Bauchdeckenreflex links oben erhalten, die übrigen $\emptyset$.

Kremaster-, Sohlenreflex beiderseits $\emptyset$.

Patellar- und Achillesreflex beiderseits $\emptyset$.

Andeutung von Babinski rechts.

Deutliche Schwäche beider Beine, besonders des rechten, Streckung besser als Beugung.

Bauchmuskulatur bei Bewegungen und Hustenstössen paretisch.

Stuhl- und Urinverhaltung.

Wirbelsäule im unteren Brust- und Lendenteil etwas prominent, druckempfindlich in Höhe des 2. und 3. Lendenwirbels.

Leichte Hypästhesie für feine Berührungsunterschiede am Innenrand der Füsse, sonst keine Sensibilitätsstörungen.

Lumbalpunktion: Normaler Druck.

Liquor gelb, gerinnt spontan, setzt nach kurzem Stehen ein Netz ab. Phase I +++ .

Lymphocytose: 625/3, im Ausstrich zahlreiche Zellen von der Grösse eines Leukocyten, rund oder mit amöboiden Fortsätzen.

Wassermann im Liquor und Blut nicht einwandfrei.

Im weiteren Verlauf ging die Facialisparese auf beide Seiten über, 
die Parese der Beine wurde total. Dann traten Gaumensegelparese links und zunehmende Schluckbeschwerden auf, so dass Patient bald nur noch mit der Schlundsonde ernährt werden konnte. Völlige Blasen-Mastdarmlähmung.

Die Sensibilitätsstörung blieb unverändert, die Reflexe an den Armen warden schwächer. Unter Erscheinungen von Herzinsuffizienz trat 8 Wochen nach Beginn der Erkrankung der Exitus ein.

Die Sektion zeigte, dass es sich um eine ausgedehnte Lymphosarkomatose des Magens and fast des ganzen Darmtraktus handelte mit Metastasenbildung in den mesenterialen und retroperitonealen Lymphdrüsen, der Leber, der Prostata, der Dura mater cerebri und der Wirbelsäule. Im 1. Lumbalwirbelkörper findet sich eine langgestreckte, unregelmässig unterbrochene, dunkelblaurote, weiche Geschwulstmasse, die vorn einen erbsengrossen Knoten bildet und hinten, am Austritt der Vena basi-vertebralis, in den Rückenmarkskanal durchgebrochen ist. An derselben Stelle ist auch im 2. Lendenwirbel eine das Lig. longitudinale posticum durchwuchernde erbsengrosse Tumormasse bemerkbar. Dementsprechend weist das Rückenmark ca. $2 \mathrm{~cm}$ oberhalb des Conus auf der rechten Seite eine $2 \frac{1}{2} \mathrm{~cm}$ lange hellgelbgraue, erweichte Partie auf. Im übrigen erschien das Rückenmark makroskopisch intakt, auch mikroskopisch zeigte der Querschnitt des Rückenmarks, und der Medulla oblongata keine Anomalie. Dagegen war die Pia mater in der gesamten Zirkumferenz diffus mit Sarkomzellen infiltriert.

Fall 23. M. K. (Prot.-Nr. 9412/1913), 26jährige, hereditär nicht belastete Fran, erkrankte vor 4 Wochen aliut mit Schmerzen in der linken Wade, die bis zur Sakralgegend ausstrahlten und allmäblich zunahmen; schliesslich wurden die Beine schwächer, sie konnte Stuhl und Urin nicht mehr halten.

Status: Grazile Frau. Innere Organe gesund.

Unterer Bauchdeckenreflex schwach.

Sphinkter- und Analreflex sehr schwach.

Unwillkürlicher Stuhl- and Urinabgang.

Ischiadicus beiderseits sehr druckempfindlich.

Patellarreflexe gesteigert, links Patellarklonus.

Achillesreflex beiderseits fehlend.

Reithosenanästhesie und Hypästhesie an der Aussenkante beider Füsse. Röntgenbefund der Wirbelsäule normal.

Lumbalpunktion: Normaler Druck. Klarer, wasserheller Liquor. Phase $\mathrm{I}++$.

Lymphocytose $\emptyset$.

Wassermann $0,2-1,0 \quad 0$.

Wassermann im Blut $\varnothing$.

Die Laminektomie, die sich vom 11. Brust- bis zum 3. Lendenwirbel erstreckte, liess keinen Tumor oder äusserlich wahrnehmbare Veränderungen der Medulla spinalis erkennen. Nach wenigen Tagen kam die Patientin zum Exitus.

Die Sektion zeigte, dass es sich um ein äusserst seltenes Krankheitsbild handelte.

An der Rückseite des Manubrium sterni, sowie auf der Vorderseite 
der mittleren Brustwirbelkörper fanden sich ausgedehnte chloromatöse Wucherungen. Ebensolche Massen lagen im kleinen Becken dem Kreuzbein an und waren durch den Sakralkanal nach oben hin vorgedrungen, so dass sie bis zur Höhe des 1. Sakralwirbels hinauf die Candafasern fest umscheideten. ${ }^{*}$ )

Endlich hatte ich vor kurzer Zeit noch Gelegenheit, den Liquor eines 22jährigen jungen Mädchens zu untersuchen, das vor 2 Jahren durch einen Sturz eine schwere Zertrümmerung des 3. und 4. Dorsalwirbels erlitten hatte. Es bestand bei ihr seitdem eine totale Anästhesie von den Mamillen abwärts mit völliger Blasen-Mastdarmlähmung und Erloschensein sowohl der Sehnen-, als auch der Hautreflexe an Abdomen und unteren Extremitäten. In diesem Falle fand sich keine Spur von Phase I; Lymphocytose bestand nicht, Wassermann im Blut und Liquor bis 1,0 war negativ.

Wahrscheinlich hatte hier das Trauma eine totale Zermalmung des Rückenmarksquerschnittes bewirkt, während die Liquorzirkulation möglich blieb oder sich allmählich wieder herstellte. Auch kommt ja hier infolge der Akuität des Traumas die Rückenmarksschädigung unter ganz anderen Verhältnissen zustande, als bei Tumoren oder dergleichen. AnderweitigeBeobachtungen über Liquorveränderungen bei Wirbelsäulenfrakturen scheinen bisher nicht gemacht zu sein.

Nach dieser Aufzählung unserer neuen Fälle möchte ich nunmehr zunächst über einschlägige Mitteilungen anderer Autoren berichten, die mir bei meiner ersten Arbeit entgangen waren oder nach ihrer Publikation veröffentlicht worden sind.

Aus dem Jahre 1897 bereits datiert eine Mitteilung von Busch (1), der bei der Obduktion eines 9 jährigen Knaben mit diffusem Angiosarkom der Leptomeningen von Hirn und Rückenmark die stark erweiterten. Seitenventrikel von gelblich-weisslicher Flüssigkeit erfüllt fand. Der Aquädukt war durch Tumormassen verlegt.

Bei einem 32jährigen Mann mit hämorrhagischem Gliom des rechten Pedunculus cerebri sah Erb (4) bernsteingelben, unter hohem Druck stehenden Liquor mit viel Fibrin, reichlich Albumen und vielen Erythrocyten, sowie reichlich polynukleären Leukocyten und grossen Lymphocyten. Einen klaren, leicht gelblichen Liquor ohne Albumen und mit wenig Lymphocyten fand er bei einem 45jährigen Mann mit Carcinom der oberen Halswirbel. Die Diagnose konnte leider nicht anatomisch sichergestellt werden.

Mohr (12) beobachtete bei einem langsam wachsenden extramedullären Caudatumor gelblich verfärbten, klaren Liquor ohne Eiweissvermehrung, der Cholesterintafeln und verfettete Geschwulstzellen enthielt. Der Patient musste unoperiert entlassen werden.

*) Eine ausführliche Bearbeitung dieses Falles soll demnächst erfolgen. 
Er erwähnt einen ähnlichen Fall von Léri und Catola (10), welche bei einem fast das ganze Lendenmark einnehmenden Tumor stark gelbgefärbten Liquor mit Geschwulstzellen beobachteten. Es handelte sich um ein voin Ependym ausgehendes Epitheliom bei einem 42 jährigen Manne.

Reich (14) hat sich ausführlich mit Entstehung und Bedeutung der Xanthochromie befasst. Ihre Entstehung infolge von Blutungen sieht er durch den Nachweis von Hämatinkristallen als erwiesen an. Das Wesentliche sei, dass die Tumoren irgendwie mit der Oberfläche von Hirn oder Rückenmark, auch der Ventrikel in Berïhrung stehen, also vom Liquorstrom getroffen werden, da sonst kein Blutfarbstoff in den Liquor übertreten könne. Die Lymphocytose, wie auch die Eiweissvermehrung sieht er als Reaktion auf meningeale Reizung an.

Bei 5 Fällen von Cysticerkose des Hirns und Rückenmarks fand Goldstein (7) dreimal Eiweiss- und Zellvermehrung im Liquor. Letztere fand bei späterer mikroskopischer Untersuchung ihre Erklärung in einer starken entzündlichen Infiltration der Gefässwände und der Pia.

Siemerling (17) veröffentlichte einen lange klinisch beobachteten Fall von gliösem Tumor, der in Höhe des 10. Dorsalsegments diffus begann, sich nach oben zu einem kompakten Strang mit zentraler Höhlenbildung verdichtete und als Gliastift im vierten Ventrikel endigte. Bei den ersten Lumbalpunktionen wurde eine hellgelbe Farbe bemerkt, die nach Verlauf von 2 Jahren nicht mehr nachweisbar war. Mit Magnesiumsulfat entstand eine leichte Trübung, die Lymphocytose war ganz gering. Die Gelbfärbung liess sich auf Blutungen in dem Tumor zurückführen, der an verschiedenen Stellen die Peripherie des Marks erreichte.

Zitronengelben Liquor, der beim Stehen sofort gerann, vermehrten Eiweissgehalt, keine Vermehrung der Lymphocyten zeigte, fand er bei einem Tumor im Cervikalmark. Ferner beobachtete er bei einer 49jährigen Frau mit einem Fibrom des mittleren Dorsalteils leichte Trübung mit Magnesiumsulfat ohne sonstige Veränderungen.

Eine 45jährige Frau mit einem Fibrom des oberen Dorsalteils hatte einen rostbraunen bis hellgelben Liquor, der starke Trübung mit Magnesium- und Ammoniumsulfat ergab. Ausserdem enthielt er viele Lympho- und Leukocyten.

Schliesslich führt er noch eine Beobachtnng von Flatau (5) an, der bei einem 40 jährigen Mann mit malignem Caudatumor zitronengelben, bald gerinnenden Liquor sah. Die Menge der Chloride war vermehrt bei sehr wenig Eiweiss und Abwesenheit von Blutfarbstoff. 
Einen Fall, der, wie die mikroskopische Untersuchung erwies, unserem Fall 14 ähnelte, beobachtete Schnitzler (16). Bei einem chondrodystrophischen Zwerg von 41 Jahren entwickelte sich in etwa 2 Jahren ein Krankheitsbild, das an einen Tumor in Höhe des 1. Lumbalsegments denken liess. Unter sehr geringem Druck entleerte sich eine kleine Menge intensiv zitronengelber Flüssigkeit. Phase I war sofort lebhaft, daneben bestand unzweifelhafte Lymphocytose; eine exakte Zellzählung war aus äusseren Gründen nicht möglich. Bei der Sektion fand sich eine zirkumskripte Vereiterung an der Cauda, die als Folge der Lumbalpunktion angesprochen wurde. Abgesehen hiervon war in der Höhe des 2. Lưmbalsegments, wo der Tumor angenommen war, das Rückenmark auf 2-3 mm Länge tief eingeschnürt, ohne dass sich äusserlich eine Erklärung hierfür fand. Die genaue mikroskopische Untersuchung ergab eine funikuläre Myelitis im Sinne Hennebergs. Die Gelbfärbung des Liquor sieht Schnitzler nicht als direkte Folge von Hämorrhagien an, vielmehr rechnet er den Farbstofi zur Gruppe der Luteine.

Greenfield (8) beschrieb Liquorveränderungen bei drei Tumorfällen. Bei einer Carcinomatose der Meningen war der Liquor gelb, klar, sehr eiweissreich und enthielt nur wenige Zellen. Ein klarer, farbloser Liquor bei metastatischem Sarkom in den Meningen enthielt 1,1 Proz. Alb. nach Noguchi ohne Zellvermehrung. In dem dritten Fall - es handelte sich um ein 15jähriges Mädchen mit subakut entstandener Paraplegia spastica inferior, Blasen-Mastdarmlähmung und Anästhesie bis zum 9. Dorsalsegment - war der Liquor klar und farblos, der Zellgehalt nicht vermehrt, dagegen fanden sich 2,2 Proz. Alb. nach Noguchi. Die klinische Diagnose: Kompression der Medulla durch Tumor konnte nicht bestätigt werden, da die Laminektomie verweigert wurde. Greenfield erwähnt dann noch einen Fall von Cooper (2), der gelblichbraunen, wie Pleurapunktat oder Ascites aussehenden Liquor sah. Noguchi und Phase I waren stark positiv, ergaben einen dicken Niederschlag, der Zellgehalt war nicht vermehrt. Die Operation zeigte, dass es sich um ein Angiosarkom handelte mit ausgesprochenem Ödem der Meningen distal von der Kompressionsstelle.

Ebers (3) fand bei einer jungen Frau mit den Symptomen von Querschnittsmyelitis einen deutlich gelb gefärbten Liquor mit starkem Eiweissgehalt, starker Phase I und wenig Leukocyten. Die von Garré vorgenommene Operation zeigte in Höhe des 1.-3. Brustwirbeldorns ein extra- und intradural gelegenes grosszelliges Sarkom, das zapfenförmig die Medulla arrodiert hatte.

Zwei intradurale Neurofibrome sah Jancke (9). Das erste Mal 
lag der Tumor in Höhe des 9. Dorsalsegments, der Liquor war klar, bernsteingelb, wurde schnell gallertig, gab sehr starke Phase I bei 20 Zellen im $\mathrm{cmm}$. In dem zweiten Fall, in Höhe des 4.-5. Dorsalsegments, war der Liquor farblos, klar, Phase I sehr stark, nur 9 Zellen im $\mathrm{cmm}$. Beide Male liess sich der Tumor exstirpieren, doch gingen die Patienten an sekundärer Meningitis zugrunde.

Multiple, gestielte Fibrome auf dem Cervikalmark fanden Merzbacher und Castex (11) bei einem Manne, der seit 18 Jahren allmählich zunehmende Lähmungserscheinungen aufwies. Der klare Liquor ergab schwach positive Eiweissreaktion und leichte, kaum pathologische Lymphocytose. Die Cholinprobe war positiv.

Unsere 8 neuen Fälle einbegriffen sind also 24 weitere Beobachtungen gemacht über Liquorveränderungen bei Rückenmarkskompression. Ich lasse zunächst umstehend wieder eine kurze tabellarische Übersicht folgen.

Bei einem Überblick über das ganze vorliegende Material fällt sogleich die grosse Verschiedenheit der Kompressionsursachen auf, die zu Veränderungen des Liquor cerebrospinalis geführt haben. Ebenso können ganz gleichartige Liquorveränderungen durch Schädigung ganz verschiedener Abschnitte der Medulla hervorgerufen werden. Dass deshalb der Liquorbefund allein keine diagnostischen Schlüsse auf Art und Lokalisation der Kompression zulässt, ist schon wiederholt, so auch von mir in meiner vorjährigen Arbeit, hervorgehoben worden. Dies gilt auch für die Xanthochromie, die Klieneberger noch als charakteristisch für raumbeschränkende Prozesse in den unteren $A b-$ schnitten des Wirbelkanals ansprach, die seither aber auch bei Kompression der höheren Partien, sogar des Halsmarks, beobachtet ist (Siemerling, Ed. Schwarz, Grund, Schnitzler II).

Eine Frage, die bisher wenig beachtet zu sein scheint, ist die, ob die Liquorveränderungen differieren, je nachdem das komprimierende Moment (I.) extradural, (II.) intradural, aber extramedullär oder (III.) intramedullär gelegen ist. Das mir aus eigener Beobachtung an Nonnes Material oder aus der Literatur zugängliche Material ergab Folgendes:

I. Extradurale Kompressionsursache:

a) Isolierte Phase I: 9 Fälle von Spondylitis tuberculosa (Schnitzler III-VI, Henkel I und II, unsere Fälle 6, 19, 20).

3 Sarkommetastasen (Nonne III und unsere Fälle 4 und 10). 
Weitere Beiträge zur Kenntnis des Kompressionssyndroms usw.

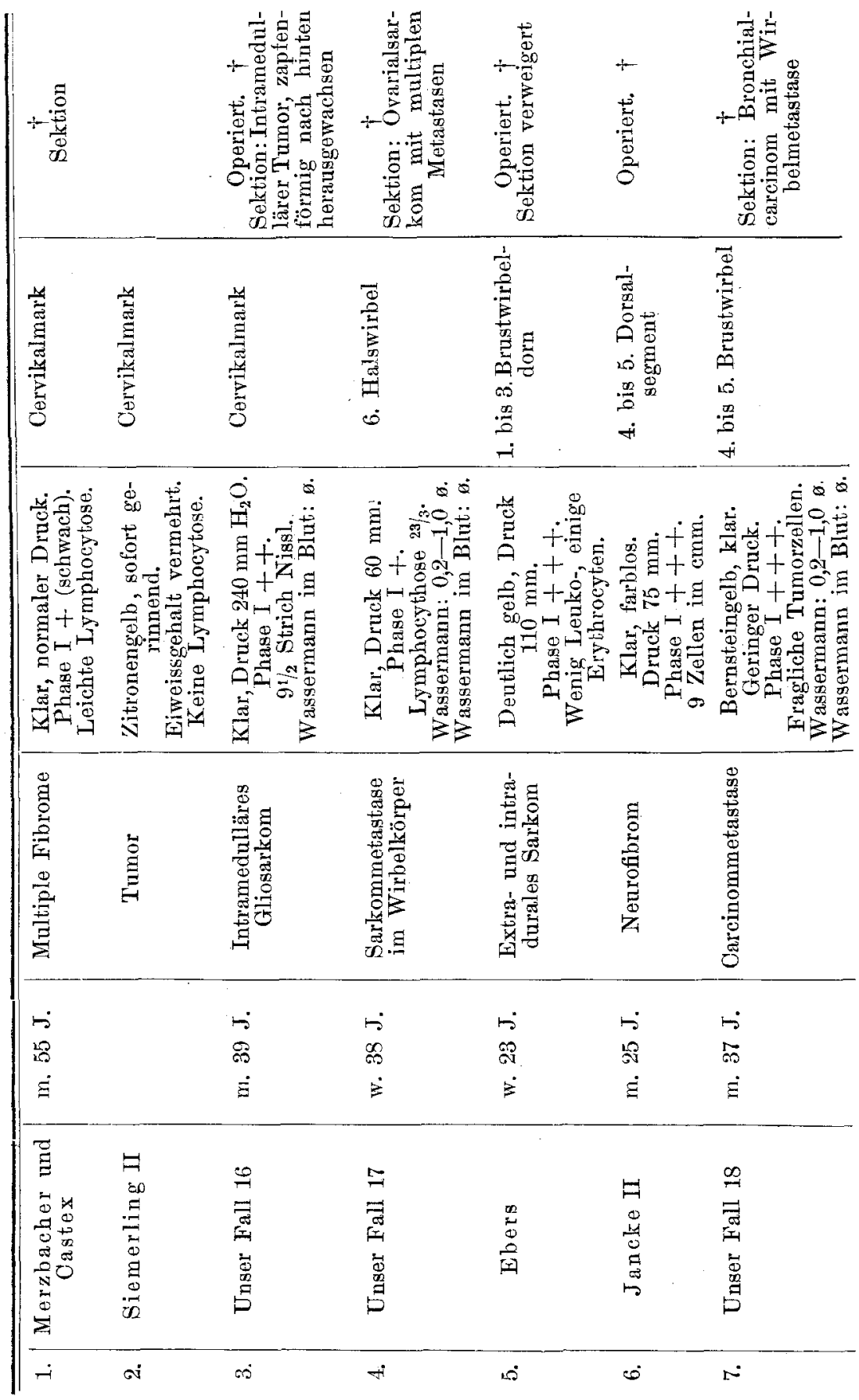




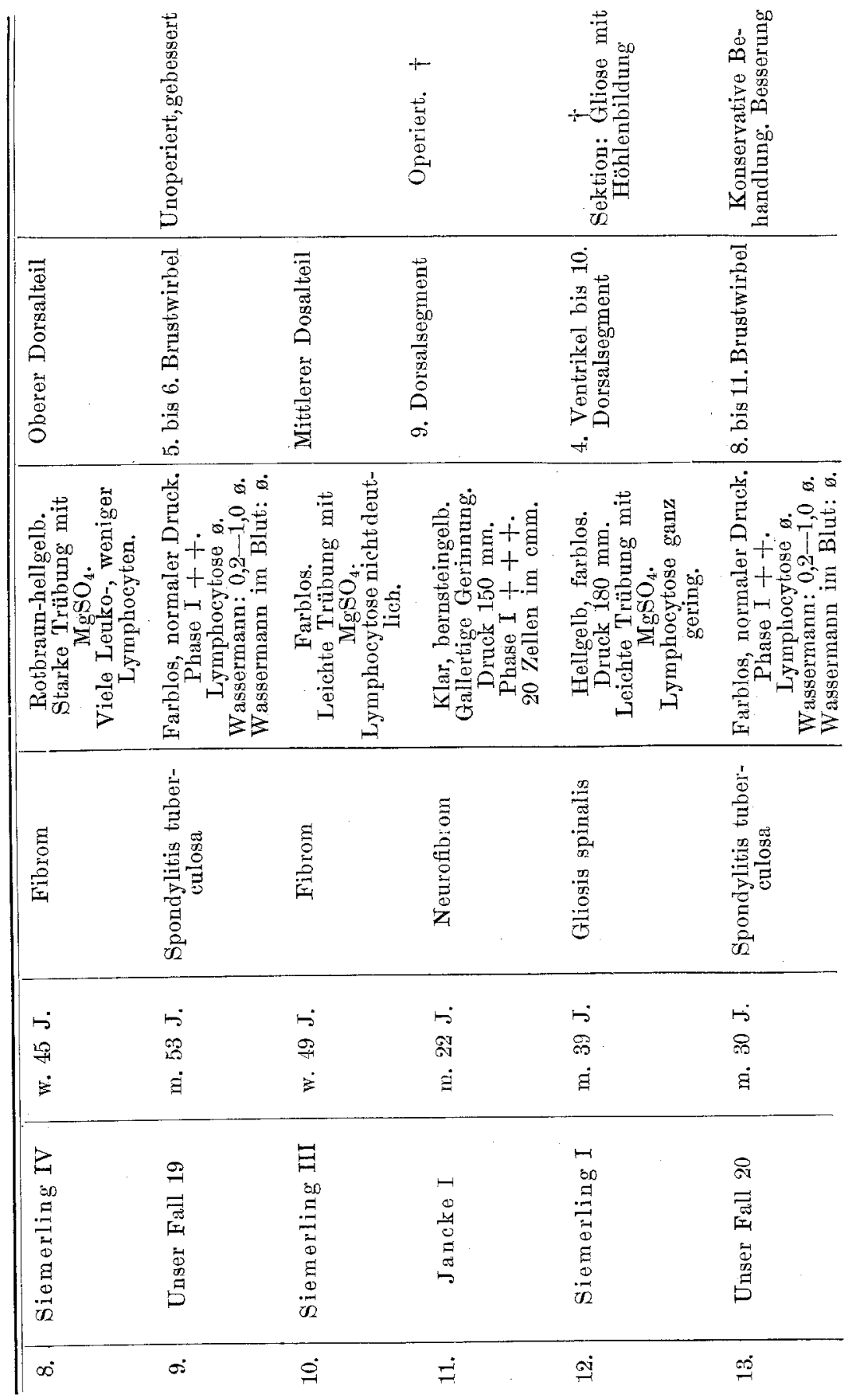


Weitere Beiträge zur Kenntnis des Kompressionssyndroms usw.

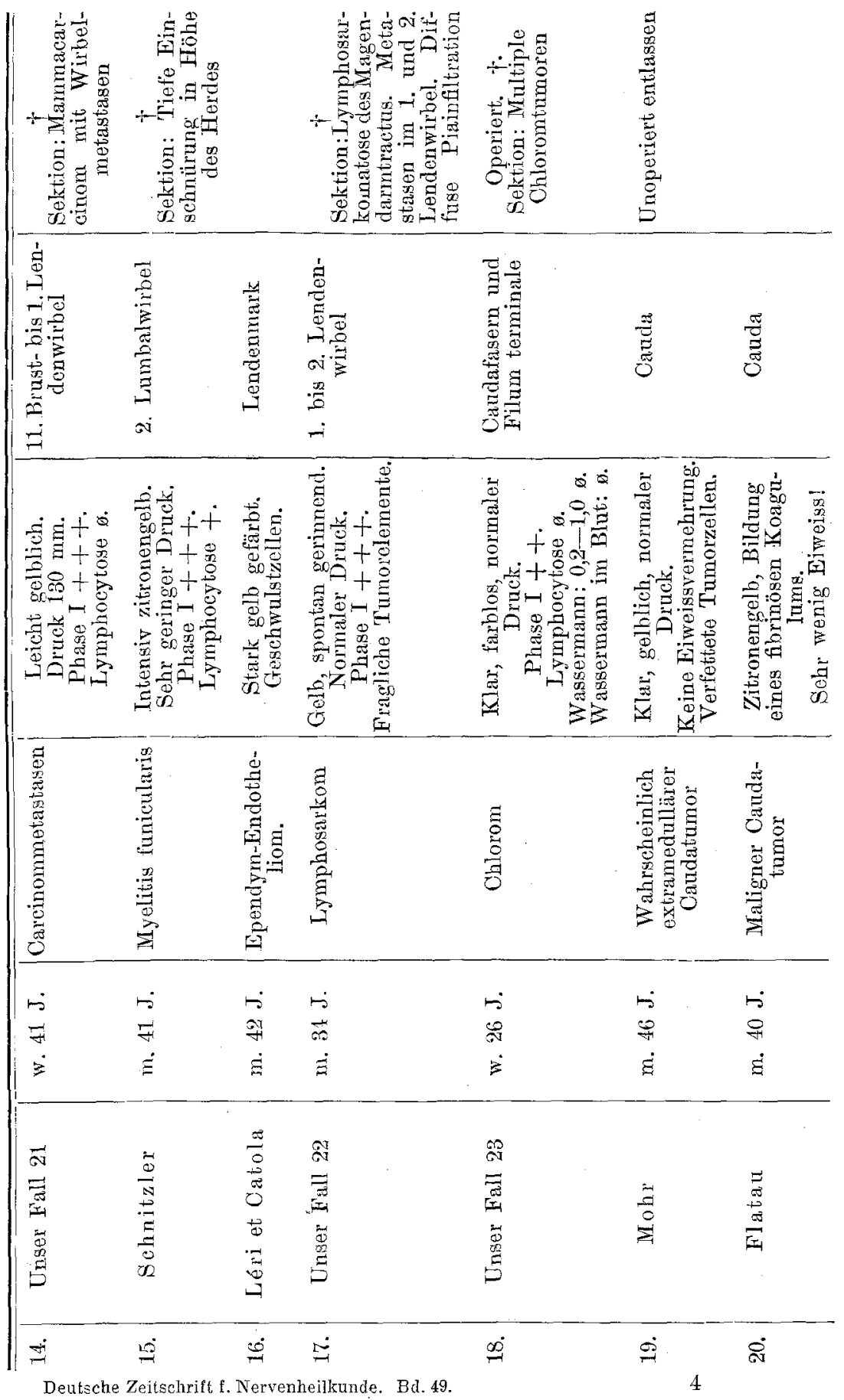




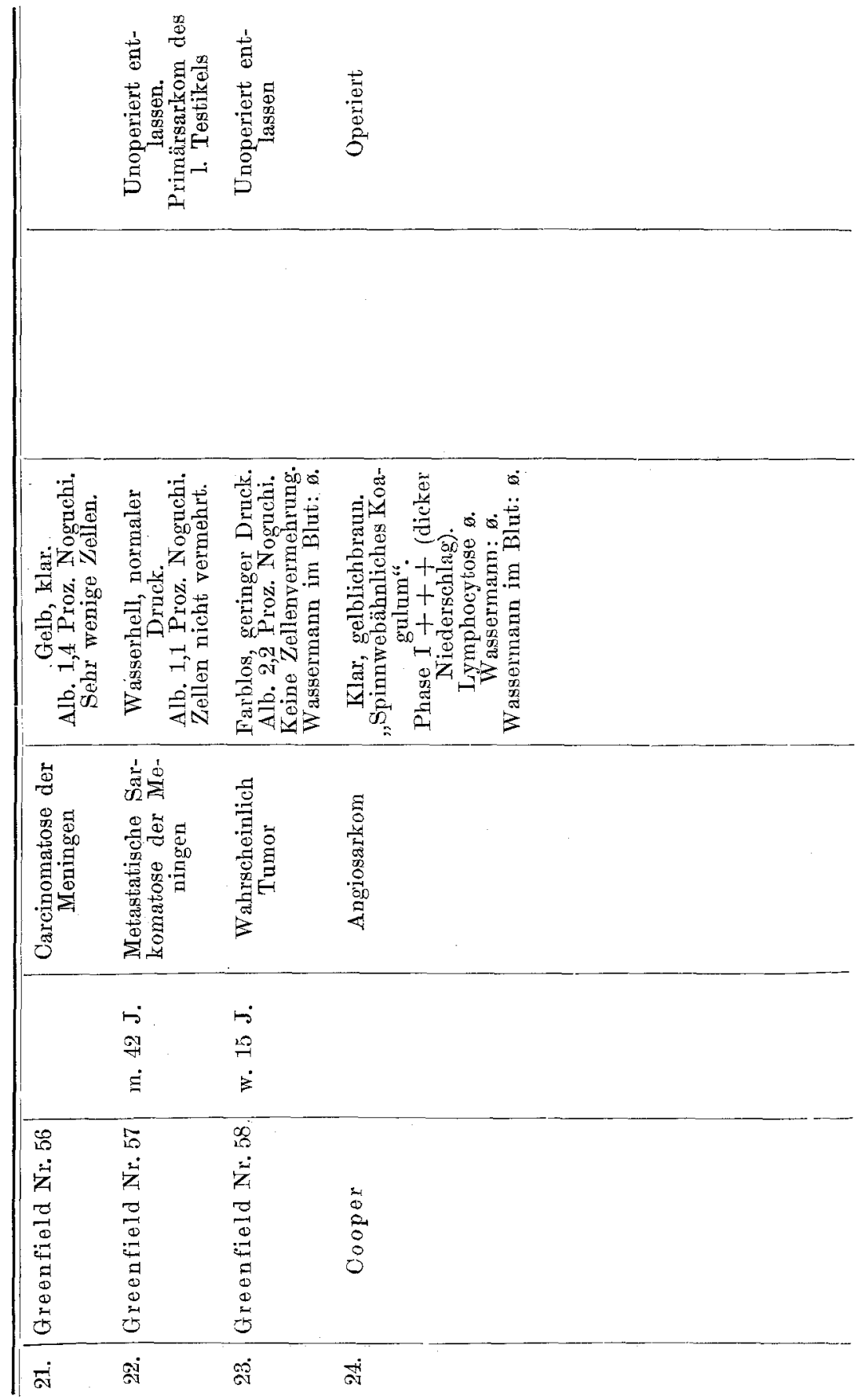


2 Chlorome (Beltz und unser Fall 23).

1 Osteom (unser Fall 8).

b) Phase I + leichte Lymphocytose: 1 Sarkommetastase (anser Fall 17).

c) Xanthochromie mit Zellvermehrung: 2 Spondylitis tuberculosa (unsere Fälle 7 und 9).

1 Carcinommetastase (unser Fall 21).

d) Xanthochromie mit Zellvermehrung: Spondylitis tuberculosa (Heilig II).

Sarkom- resp. Carcinommetastase (Quincke und unser Fall 18).

II. Intradurale, extramedulläre Kompression:

a) Isolierte Phase I: 3 Fibrome (unser Fall 11, Merzbacher, Jancke II).

1. Solitärtuberkel (Bönniger und Adler).

1 diffuse Sarkomatose (Rindfleisch II).

b) Xanthochromie ohne Zellvermehrung:

1 Gliosarkom (Ed. Schwarz).

1 Angiosarkom (Cooper).

2 Sarkome der Dura resp. Pia (Blanchetière et Lejonne und Schnitzler I).

c) Xanthochromie mit Zellvermehrung:

2 Fibrome (Klieneberger I und Jancke I).

1 Caudatumor ohne nähere Bezeichnung (K'lien e berger II). 1 mal Arachnoidealcysten mit entzündlicher Piainfiltration (Klieneberger IV).

1 Meningitis serosa circumscripta (Fumarola und Tramonti).

2 Sarkomatosen der Meningen (Rindfleisch I u. III).

III. Intramedulläre Kompression:

a) Isolierte Phase I:

3 Gliome resp. Gliosarkome (Andernach, Nonne VI, unser Fall 16).

b) Xanthochromie ohne Zellvermehrung:

Gliosis (Si emerling).

Gliosarkom (Grund).

In einigen Fällen war der Sitz der Kompression sowohl extrawie auch intradural. Bei Nonne II und unserem Fall 5 (Fibrosarkom resp. Hodgkintumoren) fand sich nur Eiweissvermehrung bei wasserhellem Liquor; unser Fall 22 und der von Ebers (Lympho- 
sarkom resp. Sarkom) zeigten deutliche Xanthochromie mit intensiver Phase I.

Die Neurofibrome in dem Fall von Reichmann and Röpke sassen teils extra-, teils intramedullär. Dex Liquor war zitronengelb, enthielt 4,6 Proz. Alb. ohne Iymphocytose.

In den beiden Fällen ron Myelitis funicularis (Schnitzler und unser Fall 14) war der Liquor zitronen- bis bernsteingelb mit starkerPhase I. Bei Schnitzler bestand auch Zellvermehrung.

Auch bei dieser Betrachtung zeigen also die Liquorveränderungen eine grosse Mannigfaltigkeit, und es ergeben sich keine typischen Unterschiede bei den verschiedenen Ursachen und Lokalisationen der Kompression. Bemerkenswert ist jedenfalls die Tatsache, dass unter dem bier untersuchten Material bei extraduraler Kompressions. ursache sich nur in 8 von 24 Fällen Xanthochromie fand, d. h. in 33,3 Proz. Dagegen war sie in 68,75 Proz., nämlich bei 11 von. 16 Fällen mit intraduralem Sitze der Kompression nachweisbar.

Wo ausgesprochene Lymphocytose bestand, war sie meistens aut entzündliche Veränderungen oder auf Beimengung von Tumorelementen zurückzuführen.

Nach den bisherigen Erfahrungen lassen also die geschilderten Liquorbefunde - isolierte Phase I oder Xanthochromie mit Eiweissvermehrung - bei Ausschlnss anderer Krankheitsursachen nur die Deutung zu, dass eine Kompression des: Rückenmarks vorliegt; dagegen geben sie keinen Fingerzeig binsichtlich der Art, der Ursache oder dex Lokalisation. dieser Kompression. Weiteren Beobachtungen muss es vorbehalten bleiben, wenn möglich anch hierin neae Fortschritte zu bringen.

\section{Literatur.}

1) Busch, Ein Fall von ausgebreiteter Sarkomatose der weichen Häutedes zentralen Nervensysiems. Deutsche Zeitschr. f. Nervenheilkunde. 1897. 9. 114 .

2) Cooper, Journ. of the Americ. Med. Ass. 1610. 2298.

3) P. Ebers, Fall von operiertem Rückenmarkstumor. Deutsche med. Wochenschr. 1913. 39. 70.

4) Erb, Über die Diagnose und Frühdiagnose der syphilogenen Erkrarakungen des zentralen Nervensystems. Deutsche Zeitschr. f. Nervenheilkunde. 1907. 33. 425 .

5) Flatau, Über Xanthochromie und Bildung eines fibrinösen Koagulum im Liquor cerebrospinalis. Neuroglia polska 1910. 6. (Ref. im Jahresber. f: Neurol, u. Psych. 1910. 423.\} 
Weitere Beiträge zur Kenntnis des Kompressionssyndroms usw.

6) Geissler, Über Blut in der Spinalflüssigkeit. Münchn. med. Wochenschr. 1913. 121.

7) K. Goldstein, Ein Beitrag zur Lehre von der Gysticerkose des Gehirns und Rückenmarks, insbesondere der Meningitis cysticercosa. Arch. f. Psych. u. Nervenkrankh. 1912, 49. 742.

8) Greenfield, On the value of a quantitative albumin estimation of the cerebro-spinal fluid. (With special reference to the syndrome of massive coagulation and xanthochromia.) The Lancet. 1912, II. 90. Jahrg. 685.

9) Jancke, Beitrag zur Diagnostik der Rückenmarkstumoren. Münchn. med. Wochenschr. 1913. 1033.

10) Léri é Catola, Epithéliome de la moelle. Revue neurologique. 1905. 768.

11) Merzbacher u. Castex, Über ein sehr grosses multilobuläres Fibrom im Cervikalmark. Deutsche Zeitschr. f. Nervenheilkunde. 1913. 46. 146.

12) Mohr, Zur Pathologie des Liquor cerebrospinalis. Deutsche Zeitschrift f. Nervenheilkunde. 1912. 44. 417.

13) H. Oppenheim, Diagnose und Behandlung der Geschwülste innerhalb des Wirbelkanals. Deutsche med. Wochenschr. 1909. 35. 44.

14) J. Reich, Über Gelbfärbung der Cerebrospinalflüssigkeit. Mitteilnng a. d. Grenzgeb. d. Med. u. Chir. 1912. 25. H. 4.

15) Reichmann, Über einen operativ geheilten Fall von mehrfachen Rückenmarksgeschwülsten bei Recklinghausenscher Krankheit nebst Bemerkungen über das chemische und cytologische Verhalten des Liquor cerebrospinalis bei Gehirn- und Rückenmarksgeschwülsten. Deutsche Zeitschrift f. Nervenheilkunde. 1912. 44. 95.

16) Schnitzler, Klinische Beiträge zur Kenntnis der mit Muskelatrophien verlaufenden Formen von multipler Sklerose und chronischer Myelitis. Zeitschrift f. d. ges. Neur. u. Psych. 1912. 12. 310.

17) Siemerling, Gliosis spinalis und Syringomyelie. Arch. f. Psych. u. Nervenkrankh, 1913. 50. H, 2. 